\title{
Individualization of Products and Services with Artificial General Intelligence and General Collective Intelligence
}

\author{
Andy E. Williams ${ }^{1, *}$ \\ ${ }^{1}$ Nobeah Foundation, Nairobi, Kenya
}

\begin{abstract}
INTRODUCTION: With advances in big data techniques having already led to search results and advertising being customized to the individual user, the concept of all products and services being customized for each individual is considered. Whether an online education designed solely for an individual, or the concept of online news or entertainment media, or any other virtual service being designed uniquely for each individual, designing products or services that maximize user outcomes as opposed to services that maximize outcomes for the entity providing them, or products that maximize outcomes for the entity manufacturing them, requires modeling user processes and the outcomes they target. And it requires a context aware system of cognitive computing to make use of that information to individually customize products and services.

OBJECTIVES: To explore the use of Human-Centric Functional Modeling (HCFM) to define the context of use of every product or service and the use of Artificial General Intelligence (AGI) and General Collective Intelligence (GCI) to create awareness of that context in order to automate the customization of every product or service for every individual.

METHODS: An analysis is performed to assess how and whether intelligent agents based on some subset of functionality required for AGI might be used to optimize for the individual user. And an analysis is performed to determine whether and if so how GCI might be used to optimize across all users.

RESULTS: AGI and GCI create the possibility to individualize products and services, even shared services such as the Internet, or news services, so that every individual sees a different version.

CONCLUSION: The conceptual example of customizing a news media website for two individual users of opposite political persuasions suggests that while the overhead of customizing such services might potentially result in massively increased storage and processing overhead, within a network of cooperating services in which this customization reliably creates value, this is potentially a significant opportunity.
\end{abstract}

\section{Keywords:}

General Collective Intelligence, Human-Centric Functional Modelling, Artificial General Intelligence, customization, individualization, products, services

\section{Introduction}

Individuals can be assumed to interact with products or services to in some way improve some outcome they have targeted. In order to optimize outcomes for each individual, it is important to know the outcome they were targeting, and it is important to know the fitness of all possible products or services in achieving that outcome in the current context so that the most fit might be offered to the user. Since all possible products and services includes customizations of current ones, the problem of achieving optimization of outcomes for each individual is also the problem of optimally customizing each product or service for each individual.

\footnotetext{
"Corresponding author. Email: awilliams@nobeahfoundation.org
} 
The problem of individualizing products or services is not just one of the ability to individually customize in a financially feasible way. Many products or services are already individually customized. The problem is also the limit in the ability to use all existing reasoning and information to define the problem of individual customization, and the limit in the ability to use all existing reasoning and information to formulate a solution. This includes the limit in the ability of individual users to communicate their desired outcomes and to review all possible solutions and assess which is better suited to optimally achieve those outcomes, and in addition the limit in ability to assess what choice of outcome they should optimally target. As an example of individual customization being critical to optimization of individual outcomes, assume there are currently four hundred economically viable choices of career in a village. A career selection counselling service for students should then select the best one for each individual based on all available information in real time. If the optimal career for a given youth in a given location at a given point in history is to be a carpenter, then the optimal education service is one that leverages all available teaching information in real time to teach carpentry with the teaching style best suited for that individual.

Human Centric Functional Modelling (HCFM) [1] can potentially represent any dynamical system with repeatable states in terms of the minimal set of reversible functions required to enter those states. The states accessed through these functions form a "functional state space" through which the system moves. By modelling all of the system's functions, this approach can potentially be used to represent all the behaviour of complex systems even where the mechanisms by which that functionality are implemented are unknown. From the functional modelling perspective, the functional state space of human cognition is a space of concepts or a "conceptual space". Reasoning, including the reasoning by which any product or services is used, is a path through that conceptual space. The task of optimizing any product or service from this point of view is the task of determining the current position of the user in their conceptual state space and the problem of determining their intended path with that product or service. Or in other words, to understand the concept of what the user is trying to achieve, and to understand the reasoning by which they intend to manipulate some product or service to achieve it.

\section{Related Work}

The functional modelling approach referred to in this paper has been used to define what is believed to be the first model of Artificial General Intelligence or AGI [3] with the potential capacity for human-like general problem-solving ability, as well as a model for General Collective Intelligence or GCI [6], [7] with the potential for exponentially greater general problem-solving ability (intelligence) than any individual. Though others have described the concept of a general collective intelligence factor (c factor) [3], though at least one other has defined a model for a general collective intelligence factor [4], and though others have described the concept of a collective super intelligence [5], to the author's knowledge the author's own work is the only model that defines the specific mechanisms required for a GCI with the capacity to achieve super intelligence in that general collective intelligence factor. Because of this, and because this model is so new, all of the existing literature surrounding GCI refers to the author's own published work, or pre-prints of the author's unpublished work that are still under review.

\section{Purpose, Research Questions, and Approach}

Human-Centric Functional Modeling (HCFM) can be used to define functional state spaces within which human processes are well-defined paths, and can be used to define a functional state space for every product or service in which every use of that product or service is a path through that functional state space. The problem of achieving any specific outcome that might be targeted through the use of any product or service can be represented as a specific navigation problem through the functional state space of the product 
or service, as well as a specific navigation problem through the functional state space of the user's cognitive system in the process of using that product or service (i.e. the path through the user's conceptual space). Therefore by considering any given individual's desired paths through their conceptual space, and by considering the available paths through the functional state space of the product or service, it is possible to automate the customization of those products and services for that individual. The purpose of this paper was to demonstrate the capacity of functional modeling to define a context of execution for every functionality of every product or service, and demonstrate the capacity of functional modeling together with Artificial General Intelligence and General Collective Intelligence to significantly increase capacity to individualize the execution of every product or service in order to significantly increase any targeted collective outcomes of their execution through maximizing targeted outcomes for every individual.

The research questions addressed in this paper and the method with which they were approached are described below:

\section{Section 3: Research Question Addressed}

3.1 Can functional state spaces defined within a Human-Centric Functional Modelling approach be used to define the individualization required to optimize a product or service for any individual user or group, and can AGI and GCI be used to automate the customization of those products and services for that individual or to groups of individuals?

\section{Section 4: Model}

4.1. Defining a universal representation for information and collective reasoning.

4.2. Defining the functionality required for collective reasoning.

\section{Section 5. Method}

5.1. An analysis is performed to assess how functional models might be individualized.
5.2. An analysis is performed to assess whether the truth of individualization requirements might be determined.

5.3. An analysis is performed to assess whether individualization might be optimized according to those requirements.

\author{
Section 6. Findings \\ Section 7. Research Limitations \\ Section 8. Practical Implications \\ Section 9. Conclusions
}

\section{Model}

A full discussion of the functional modelling approach is too broad for a single paper. Aspects of the approach that are relevant to the research questions are described below.

\subsection{Functional Modelling of Products or Services and Customization and AGI}

From the functional modeling point of view, any reasoning can be represented in conceptual space as a process or function that receives a set of concepts as its inputs, that produces a set of concepts as its outputs, and that depends on other concepts that form its "context of execution". In the case of reasoning processes multiple inputs might be received at multiple different times to produce outputs at multiple different times.

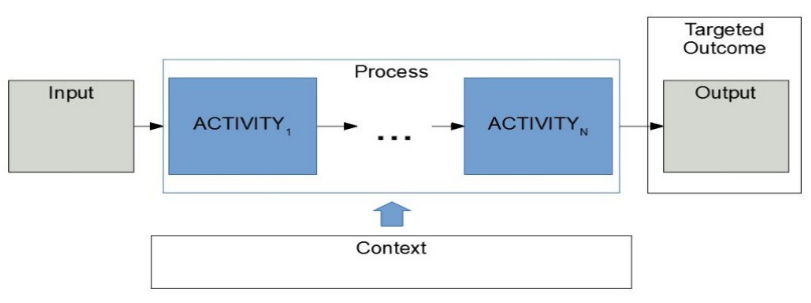

Figure 1. Functional model of processes and their context of execution.

And any sequence of reasoning can be represented as a path through conceptual space that consists of such reasoning processes or functions. 


\section{Reasoning Path Through Conceptual Space}

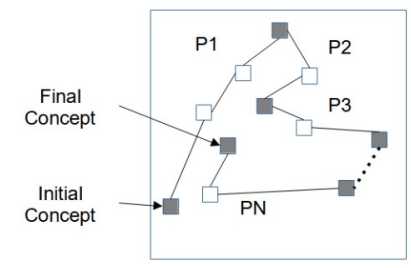

Figure 2. Reasoning is a path through the conceptual space.

Therefore any reasoning which a user might execute to use a product or service (i.e. any "usage process") can be represented by a path in conceptual space. This usage process again takes concepts as its inputs, produces concepts as its outputs, and depends on other concepts that form the "context of execution" of the process. An example of the "context of execution" of a function are the parameters $a$ and $b$ in the function $\mathrm{F}=\mathrm{ax}+$ by. In this equation the inputs are the values of the variables $x$ and $y$.

Similarly, any customization process might be represented by a path through the conceptual space of the designer (the customizer). Customization processes can potentially belong to two categories corresponding to the two types of reasoning that human beings have. Human cognition exhibits type 1 (intuitive) reasoning to detect patterns where past experience has trained the intuition. Type 1 reasoning can address uncomputable problems by detecting patterns that allow problems to be solved by observing past solutions, where the solutions cannot be calculated. And human cognition exhibits type 2 (rational methodical) reasoning to address computable problems. Accordingly, while any customization must be represented in conceptual space by inputs, outputs, and a context of execution, a customization process might be defined using intuitive reasoning with an open set of parameters that differs subjectively for each designer, or it might be defined using rational methodical reasoning consisting of a well-defined customization process with a finite set of parameters. If any customization required falls outside of that closed type 2 process and outside of those parameters the "function" cannot be calculated and the custimization cannot be performed. However a customization based on a type 1 process is subjective and can always be performed.

However, a semantically defined generalization of a type 2 customization process must accommodate semantic models of any specific type 2 customization processes, each with any set of parameters, and therefore effectively consists of an infinite set of processes and has an infinite set of parameters.

A product or service is a tool for achieving some outcome. In order to optimize outcomes for the user, the product or service comprising the tool must be customized in a way that the process of usage yields optimal outcomes. If a generalized customization approach is used, then any specific customization process fitting within that generalization might be used. Since any process of customization might potentially be used, and since the properties of the current configuration of the tool (the product or service) are some of the inputs required to that customization process, the description of the tool must be a semantic one rather than being confined to specific parameters. Tools can also be either static entities that do not change state, or dynamic entities that change state through their various functions. Tool use can therefore be represented by either a swim lane diagram with only the user playing a role in the case of a static tool.

\section{Outcomes of Static Product or Service Usage Processes}

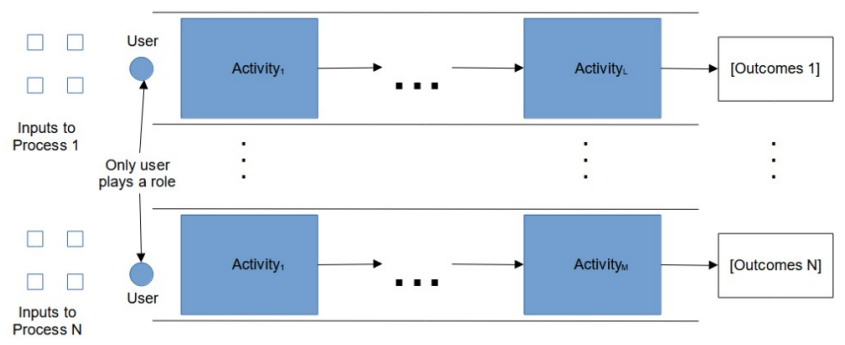

Figure 3. Usage processes for a static tool. 
Or tool use can be represented by a swim lane diagram in which the user and the tool both execute activities.

\section{Outcomes of Dynamic Product or Service Usage Processes}

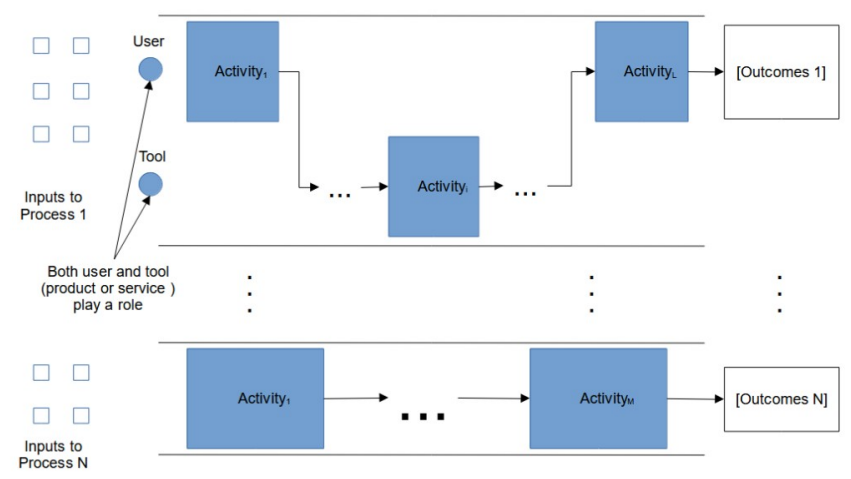

Figure 4. Usage processes for a dynamic tool.

A generalization of the user's process of usage must accommodate semantic models of any specific usage processes, each with any set of parameters, and therefore effectively consists of an infinite set of processes and has an infinite set of parameters. As mentioned, the usage process is a path through the conceptual state space of the user that takes concepts as its inputs, produces concepts as its outputs, and depends on other concepts that form the "context of execution" of the process. A generalization of the customizer's customization process must have the capacity to accept whatever usage process and whatever context of execution is defined by the conceptual space of the user. In order for the generalized usage process to be able to accommodate any possible information from the user and any possible information regarding the tool, and in order for the generalized customization process to be able to accommodate any possible information from the user, from the customizer, or about the tool, all these entities must be defined semantically as opposed to having any closed definition. And in order to be able to execute the customization process these semantic models, including the conceptual space of the user, must be available.
Obtaining all concepts from the conceptual space of the user that are relevant to the process of tool usage is akin to determining the topography of relevant parts of the user's conceptual space. The topography of the conceptual space of the user might be inferred by the experiences the user has been known to have gone through or not gone through. These experiences in turn might be inferred by the category of user, which is defined by the various properties of the user. Choosing a very simple example, a toddler might have key people and basic activities in their conceptual space.

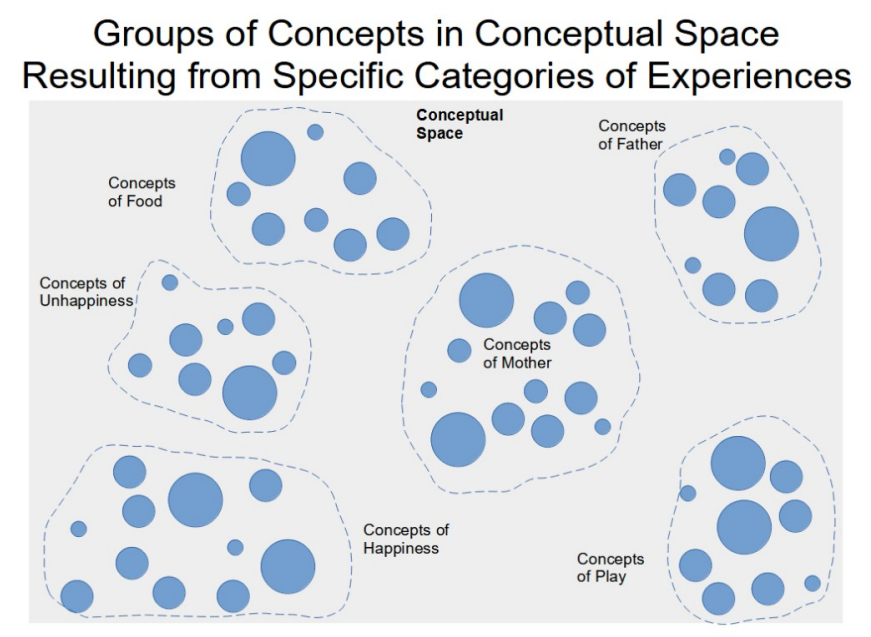

Figure 5. Conceptual space of a toddler as an example of a user's conceptual space.

The reasoning paths that might exist in this conceptual space are those that connect these concepts. 


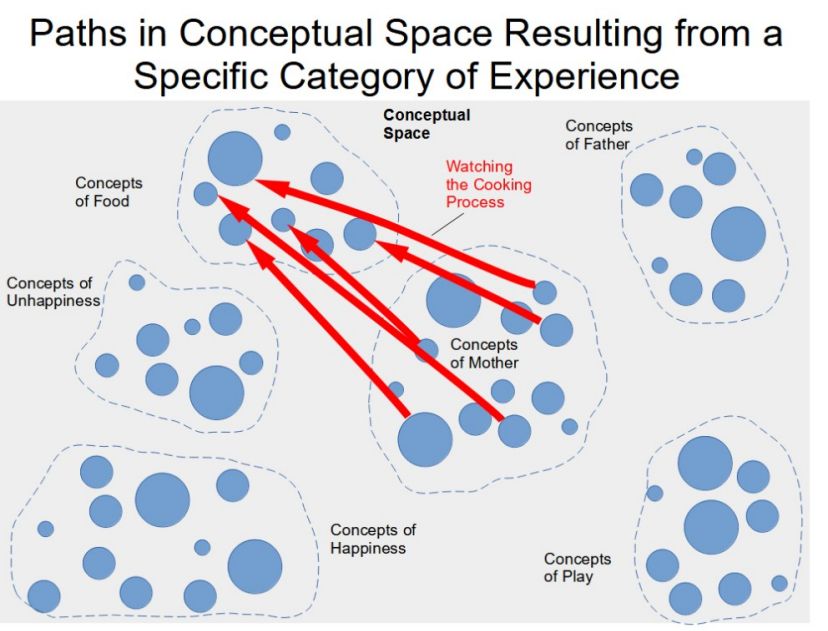

Figure 6. Reasoning paths between concepts in the user's conceptual space.

Patterns of experience might then produce patterns of reasoning paths.

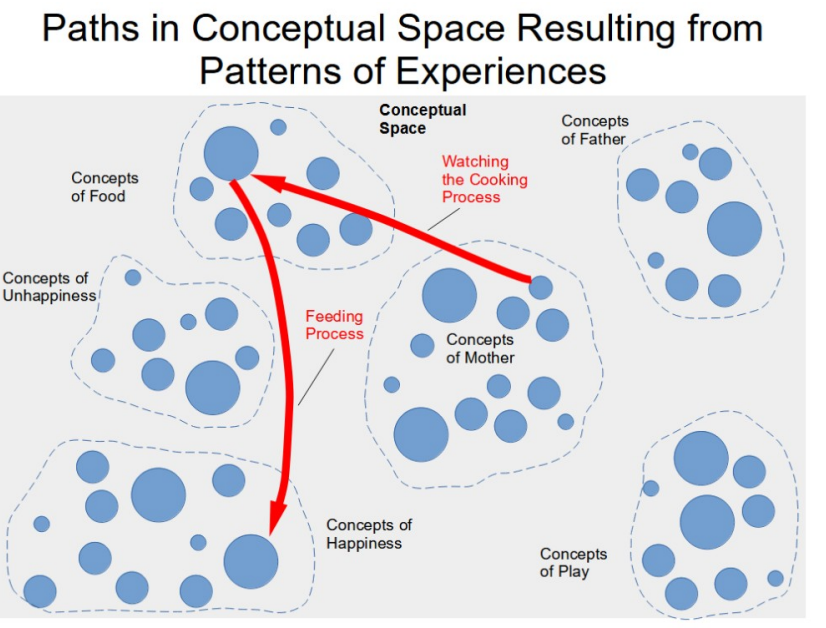

Figure 7. Reasoning paths in the user's conceptual space.

By observing a great many users of all categories, a model of the relevant concepts in the conceptual space of each user, and a model of their tool usage processes might be inferred by various combinations of testing and educated guessing. Because this is a semantic model, it might be used by any semantically defined customization process to individualize the tool for the user. All customization processes within the cognition of the designer are by definition semantically defined.

Having access to a semantic model of the relevant part of the user's conceptual space, and having a set of semantically defined customization processes for a given product or service, the cognition of the designer can then customize that product or service for that individual.

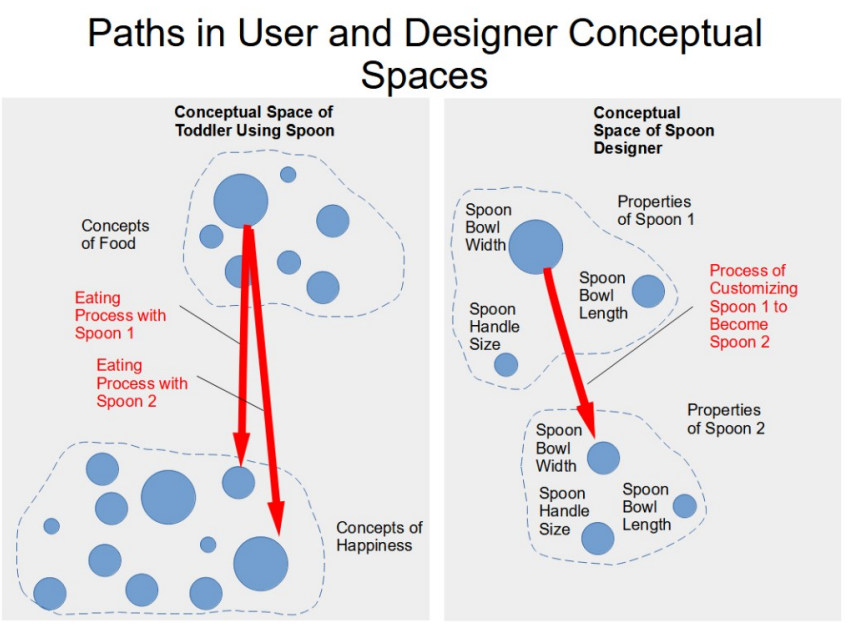

Figure 8. Usage processes in the user's conceptual space and processes to customize that usage in the customizer's conceptual space.

Products might then be individually customized by simply accessing the conceptual space of the user and accessing the conceptual space of the designer containing the customization processes, and then applying those customization processes to those usage processes in a system of artificial cognition such as an AGI. In order for individual customization of the products and services to reliably converge on a more fit configuration, the process of observation of the user's conceptual space must be accurate. However, individual users and designers might have cognitive biases that introduce systematic errors into the observation process. Fortunately, analysis of functional state spaces suggests truth potentially forms a pattern in all such spaces, including conceptual space, where this pattern, might be used in order to avoid and see through these cognitive biases [11]

In summary, written descriptions might be created for the user's usage processes, the designer's customization processes, and written descriptions of the user's or designer's understanding related to any of these. These 
descriptions might reliably convey information, but self-contained semantic descriptions are required to reliably convey understanding. A semantic model of the user's usage processes and understanding is required by the designer in order to customize product or services to optimize user outcomes. The conceptual space defined within the Functionl Modeling Framework (FMF) [13] for modeling the human system, that was in turn developed through Human-Centric Functional Modeling (HCFM) potentially provides the first complete semantic model of information and reasoning processes that might be used for this purpose.

If both the user and designer semantic models are available then the customization process can be applied in an automated way. For all potential usage models to be available there must be a process that continually maps out the user's conceptual space. For this customization process to maximize outcomes for the user rather than the product manufacturer or service provider, this process and the semantic model it creates must be decentralized so that it is within the user's sole control. Therefore, if this customization process must executed by an intelligent agent, it must be an intelligent agent within the user's sole control as well. In other words, the user's intelligent agent must be allowed to customize the product or service using any customization process it finds, and using all the information it has about the user. In the case of virtual products like software this customization involves customization of functionality and user interfaces. This in turn requires functional modeling to decompose the software domain into a set of functional operations, it requires the software to be modeled as a sequence of those operations, and it requires a library of implementations of each operation in the functional domain that the intelligent agent might select from. In the case of physical products this customization requires manufacturing equipment based on smart manufacturing technology that is able to process semantic models. For example, an automated process of building a running shoe might be customized to modify the size of some sewn in graphic. In order to be able to accommodate any type of graphic the graphic stitching operation must be implemented by a smart machine that accepts a semantic model of the graphic as its input.

\subsection{Functional Modelling of Products or Services and Customization and GCI}

Human lives are a set of paths through conceptual space. Any outcome an individual is attempting to achieve through use of a product or service can be represented as the problem in conceptual space of navigating from one point in conceptual space to another. The problem an individual chooses to solve, and the reasoning they use to solve it depends on the concepts available in their conceptual space and the available reasoning paths between them. The concepts in our conceptual space will depend on their education, their innate ability, and other aspects of their lived cognitive experience.

This sequence of paths might be nondeterministic, but there are also stable patterns determined by civilization, culture, and biology. These patterns might be highly useful in mapping out the conceptual space of each individual. For example, children in many cultures at this point in history go to school at a certain age, decide at a certain age what post-secondary (if any) studies they will pursue if they choose to do so, and make other key life decisions.

By following an individual's lived experience it might be possible to construct their conceptual space and the reasoning paths between those concepts that are available to them so that it might be possible to determine all the uses they might have for a product or service and then use that information to optimize the service for them.

By following the lived experience of all individuals, it might be possible to describe individual conceptual spaces in far greater detail. As an example, children of the same age might tend to have many of the same general concepts in their conceptual space. Children under the age of four who are wanting to use a drawing application to visualize concepts in their conceptual space will have different demands than trained illustrators, 
who will in turn have different demands that artists trained in abstract schools of art.

Collective reasoning based on a single semantic model requires General Collective Intelligence. Representing collective reasoning processes as consisting of reasoning activities executed by individuals within roles enables the most important properties of execution to be decoupled from any particular user so that outcomes might be massively scaled across many users. A very general representation of the single semantic model (the conceptual space) has been provided here. a more detailed representation of reasoninga processes and of the information passing in and out of those processes is provided by the Functional Modelling Framework (FMF) [13] which defines the building blocks that all semantic models must be comprised of from a functional modelling of view. But consideration of this level of detail is not necessary here and is outside of the scope of discussion.

Some intuitive examples of the usefulness of individual customization are a cookbook that not only knows the user's favourite recipes but that contains newly created dishes for which an understanding of that user was leveraged to predict with high certainty that the user will find those new recipes delicious. In functional state space the problem of using all information in the world about everyone's tastes to design the dish that will please the user most at a given time and at a given phase in their life is the same abstract problem as discovering a unified field theory, or designing a space ship, or developing a manufacturing process that can grow technology from plants. All are problems of expanding the regions of functional state space that are accessible and increasing the resolution of points in functional state space that can be located, and increasing the rate at which those paths can be navigated. Using the definition of intelligence in conceptual space as the volume of conceptual space that can be navigated per unit time, then every functional state space becomes a system of calculation with the potential to significantly increase intelligence in that domain.

\section{Method}

The research question was addressed using the methods summarized in table 2 .

Table 1. Research question and method.

\begin{tabular}{|c|c|}
\hline Research Question & Method \\
\hline $\begin{array}{l}\text { 1. Can functional } \\
\text { state spaces defined } \\
\text { within a Human- } \\
\text { Centric Functional } \\
\text { Modelling approach } \\
\text { be used to define the } \\
\text { individualization } \\
\text { required to optimize } \\
\text { a product or service } \\
\text { for any individual } \\
\text { user or group, and } \\
\text { can AGI and GCI be } \\
\text { used to automate the } \\
\text { customization of } \\
\text { those products and } \\
\text { services for that } \\
\text { individual or to } \\
\text { groups of } \\
\text { individuals? }\end{array}$ & $\begin{array}{l}\text { 1. An analysis is } \\
\text { performed to assess } \\
\text { how functional } \\
\text { models might be } \\
\text { individualized. } \\
\text { 2. An analysis is } \\
\text { performed to assess } \\
\text { whether the truth of } \\
\text { individualization } \\
\text { requirements might } \\
\text { be determined. } \\
\text { 3. An analysis is } \\
\text { performed to assess } \\
\text { whether } \\
\text { individualization } \\
\text { might be optimized } \\
\text { according to those } \\
\text { requirements. }\end{array}$ \\
\hline
\end{tabular}

\subsection{Individualization of Functional Models}

Assuming every product or service can be represented by a functional model, this functional model has inputs, operates within a context, and produces outputs that have projected and actual outcomes. This functional model can also be specified at different levels of detail, right down to the smallest components. Every instance of a product or service is then an implementation of that single high-level functional model or that detailed composite functional model. In order to apply automated decision-making to the customization of this functional model for the individual the decision system must have access to information specifying all possible implementations and the contexts in which each are most fit, and must have information defining the context of the user, in order to optimize the 
implementation for the user. In order to apply automated decision-making to the customization of this functional model for the group the decision system must have access to information specifying all possible instances of each implementation and their fitness in each context, and must have information defining the context of all users, in order to optimize the implementation for the group.

In order for this information and decision processes to optimize outcomes for the user rather than to optimize outcomes for the owner of the information or decision system, this decision system and this information must be managed in a way that is decentralized, peer to peer, usercentric, and massively collaborative, which are the properties of GCI.

\subsection{Determining the Truth in Assessments of Fitness}

Human cognition has the capacity for two different types of thought, intuitive (type 1) reasoning, and rational methodical (type 2) reasoning [8]. Due to fundamental biases in cognition, different individuals assess truth in the same topic in either of these two fundamentally different ways. Intuition is essentially a pattern detection system trained by patterns observed in the past. Intuitive reasoning therefore tends to evaluate truth depending on factors that create such patterns in observation, including what the in-group one is a part of thinks. Rational methodical reasoning demands the freedom to exercise any reasoning process. Depending on which method for assessing truth dominates in a group, different groups might be biased towards prioritizing individual rights and freedoms in assessing truth, or might be biased towards prioritizing assessing truth according to what the group believes to maximize outcomes for the group. This in turn has a large impact on the products and services those groups want to use and therefore consider as optimal. For example, in news services those biased towards intuitive reasoning might not want to see any news they "intuitively know" is fake. And those biased towards rational methodical reasoning might not want to be prevented from accessing any news whatsoever so they can judge the truth of it for themselves. This group might perceive being protected from accessing any "fake" news as censorship [14].

The challenge in individually customizing all products and services in a way that optimizes collective outcomes is therefore not determining what the group on average believes the optimal product or service is for a given individual. This is little different than imposing the opinions of any other centralized entity, which aligns outcomes with the interests of that entity rather than with collective outcomes. However, functional modelling provides a common mechanism for assessing truth in large volumes of information and identifying truth from the point of view of each individual's cognitive bias [11]. Using Human-Centric Functional Modeling (HCFM) all information and reasoning processes have a representation in some functional state space. Functional state spaces can be defined for any product or services domain. Or functional state spaces can be defined for any hardware or software application domain, for example the blockchain domain, or the communication domain. Combined with an intelligent agent based on some subset of functionality required by an AGI, where that agent execute operations within that domain on the user's behalf in order to optimize individual user outcomes, this becomes a cognitive computing application (e.g. a cognitive communication application, or a cognitive blockchain application). Combined with a platform such as the proposed P2PSF [10] that is based on some subset of functionality required by a GCI, where that GCI orchestrates execution of operations within that domain in order to optimize collective outcomes, this becomes a collective cognitive computing application (e.g. a collective cognitive communication application also known as a cognitive communication platform, or a collective cognitive blockchain application also known as a cognitive blockchain platform).

Representing every product or service in terms of a set of functional operations within a given 
domain, then the processes through which a product or service interacts with a user are a set of paths through the functional state space of that domain. Use of that product or service then moves that product or service along a path in functional state space. Regardless of the domain, all functional state spaces have the same rules defining what motion in that functional state space is unphysical and therefore untruthful. The pattern of untruth can then potentially be detected with pattern detection systems such as AI, so untruthful representations of which product or service are most fit can potentially be detected at much greater speed and scale.

\subsection{Optimization of Products or Services}

From the functional modelling perspective, optimization of a product or service involves finding a better set of paths the product or service might take through a more optimal functional state space. As in any other functional state space, including the conceptual space defined for the model of the cognitive system, a problem is the lack of a path from an initial functional state to the target functional state. Increasing capacity to optimize outcomes for an individual user then involves the same attributes required to increase problem-solving ability in the conceptual space or any other functional state space, namely increasing capacity for complexity in the path that can be navigated, increasing the number of paths that can be navigated, and increasing ability to sustain that navigation [7].

This functional optimization using AGI requires decoupling the problem-solving processes of the AGI from any instance of execution of any implementation of any functional component, so that the AGI might select other instances, implementations, or any other attribute where more optimal for the user. Using GCI to optimize collective outcomes for the group, this functional optimization requires decoupling the problemsolving processes of the GCI from any instance of execution of any process from any individual executing a role, or from any other attribute, so that the GCI might select other individuals, or any other attribute where more optimal for the group.

In the customization of products or services this decoupling has different meanings for services providers and their clients. One attribute that must be decoupled is ownership. This does not mean that no entity can own anything in such a model. It means ownership can be more easily decoupled to apply to a sub component of the service and more easily transferred. Rather than all components of service being limited to the one monolithic provider who owns the entire process, a service might self-assemble from the most optimal components available from all services providers.

In the case of websites for example, for services providers customizing services for their customers, decoupling services from centralized ownership means websites must keep all content and functionality required to create versions of the sites for each individual user, but must enable the individual user or an intelligent agent acting on their behalf to specify what that optimal configuration is. As opposed to the current situation in which the services provider centrally controls what configuration the user is shown through cookies the user can't utilize to optimize their own experience. For individuals customizing services for themselves, decoupling services means being able to access all information about all available implementations of a service everywhere without any centralized entity that removes such access. An intelligent agent working on behalf of the individual might then apply the individual's cognitive biases and preferences in what they want to see. As an example, some users with the cognitive bias towards wanting to see all information might prefer website functionality that maximizes discoverability of all information. Users with the cognitive bias towards wanting to see only information they are likely to consider true might prefer website functionality that maximizes discoverability of that content.

\section{Findings}

The findings were that a functional modeling based approach combined with an intelligent 
agent implementing some subset of functionality required for AGI might increase capacity to individually customize products or services in order to optimize outcomes for individual users. And that same functional modeling approach combined with GCI might increase capacity to customize products or services in order to optimize outcomes for groups of users. If AGI and GCI have the potential to exponentially increase outcomes, then this increase in capacity to individually customize products or services might be an exponential increase.

Table 3. Research Findings.

\begin{tabular}{ll}
\hline Research Question & Findings \\
\hline $\begin{array}{l}\text { 1. Can functional state } \\
\text { spaces defined within }\end{array}$ & $\begin{array}{l}\text { The answer } \\
\text { appears to be yes } \\
\text { a Human-Centric }\end{array}$ \\
$\begin{array}{l}\text { Functional Modelling } \\
\text { approach be used to } \\
\text { define the }\end{array}$ & $\begin{array}{l}\text { confirmation by an } \\
\text { actual }\end{array}$ \\
individualization & implementation. \\
required to optimize a \\
product or service for \\
any individual user or \\
group, and can AGI \\
and GCI be used to \\
automate the \\
customization of those \\
products and services \\
for that individual or \\
to groups of \\
individuals?
\end{tabular}

\section{Research Limitations}

It is outside of the scope of this paper to provide the functional models required to achieve mass individualization of products or services. A major effort to define functional models across a wide array of disciplines is required in order to do so. For this purpose a hierarchy of functional models called the eXtensible Business Modelling Framework (XBMF) is currently being conceptualized through ongoing work to represent processes all along the life-cycle of any product or service. All functional state spaces are expected to have some common abstract properties. As the model of the conceptual space of the cognitive system continues to be elaborated, this commonality should enable that effort to benefit the understanding of all other functional states spaces as well.

\section{Practical Implications}

One category of service that might be customized is the Internet [9]. Using AGI, a cognitive Internet client application might optimize the Internet for the user so that in essence each individual sees a different Internet to accommodate their cognitive biases, including a different version of every website. Given the 7.5 billion population of the earth, that is potentially 7.5 billion Internets. While the resource requirements of such an effort would seem to be unfeasible, GCI might be used to combine such services into networks of cooperation that create sufficient value to support such resources [12]. And using GCI, a cognitive Internet platform might allow users and services providers to self assemble into an optimal Internet for the group. The same cognitive Internet that might self assemble into an Internet that maximally blocks content that offends group sensibilities for a population that is biased to prioritize protecting against such content, is the same cognitive Internet that might self-assemble into an Internet that maximally achieves the conflicting goal of blocking the ability of any party to conduct pervasive surveillance and censorship.

\section{Conclusions}

Individualization of products and services requires massive functional modeling, and requires intelligent agents implementing some subset of the functionality required by an AGI, and acting on the user's behalf and in the user's sole control. This individualization also requires a GCI to ensure collective outcomes are maximized as opposed to maximizing the interests of any subset of decision-makers. But once these requirements 
have been met, individualization of products and services has the potential to significantly increase both individual and collective outcomes over

\section{Acknowledgements.}

Thanks to Julian Talev for countless discussions on the topic.

\section{References}

1 Pre-print: Williams, A. E. (2020, May 15). Use of Human-Centric Functional modelling to Maximize Convergence in Integrative Research. https://doi.org/10.31730/osf.io/jv6h8

2 Book Chapter: Williams A.E. (2020) A Model for Artificial General Intelligence. In: Goertzel B., Panov A., Potapov A., Yampolskiy R. (eds) Artificial General Intelligence. AGI 2020. Lecture Notes in Computer Science, vol 12177. Springer, Cham. https://doi.org/10.1007/978-3-03052152-3 38

3 Journal article: Woolley, Anita Williams; Chabris, Christopher F.; Pentland, Alex; Hashmi, Nada; Malone, Thomas W. (29 October 2010). "Evidence for a Collective Intelligence Factor in the Performance of Human Groups". Science. 330 (6004): 686688. Bibcode:2010Sci...330..686W. doi:10.1126/science.1193147. PMID 20929725. S2CID 74579.

4 Journal article: Krafft, P.M. (2019), A Simple Computational Theory of General Collective Intelligence. Top Cogn Sci, 11: 374-392.

https://doi.org/10.1111/tops.12341.

5 Book: Malone, T. W. (2018). Superminds: The surprising power of people and computers thinking together.

6 Conference: The Relationship Between Collective Intelligence and One Model of General Collective Intelligence, Andy E. Williams, Computational Collective Intelligence, 11th International Conference, ICCCI 2019, Hendaye, France, September 4-6, 2019, Proceedings, Part II, Pages 589600 those possible today, removing current limits to achieving collective well-being.

7 Pre-print: Williams, A. E. (2020, December 2). General Collective Intelligence vs the Innate Collective Intelligence Factor. https://doi.org/10.31730/osf.io/kp3x8

8 Book: Kahneman, Daniel. Thinking, Fast and Slow. New York: Farrar, Straus and Giroux, 2011.

9 Pre-print: Andy E. Williams, The Role of Cognitive Computing and Collective Cognitive Computing in the Future Internet, under review (2020).

10 Pre-print: Williams, A. E. (n.d.). The Peer to Peer Social Fabric as a Platform for General Collective Intelligence. Retrieved from osf.io/preprints/africarxiv/qbxfr

11 Pre-print: Andy E. Williams, Detecting Truth in Conceptual Space with Artificial General Intelligence and General Collective Intelligence, working draft (2020)

12 Pre-print: Williams, A. E. (2020, December 16). Discovering and Implementing Self-Sustaining Networks of Cooperation with General Collective Intelligence. https://doi.org/10.31730/osf.io/ $\underline{\text { safxk }}$

13 Pre-print: Williams, A. E. (2020, April 16). A Human-Centric Functional Modeling Framework for Defining and Comparing Models of Consciousness and Cognition. https://doi.org/10.31234/osf.io/94gw3

14 Pre-print: Williams, A. E. (2020, November 17). Bridging the Left - Right Political Divide with Artificial Intelligence and Collective Intelligence. https://doi.org/10.31730/osf.io/y65x4 